\title{
Experimental Verification of Fluctuation Relations with a Quantum Computer
}

\author{
Andrea Solfanelli, ${ }^{1,{ }^{*}}$ Alessandro Santini $\odot,{ }^{1, \dagger}$ and Michele Campisi $\odot^{2,3, \ddagger}$ \\ ${ }^{1}$ SISSA, via Bonomea 265, Trieste I-34136, Italy \\ ${ }^{2}$ NEST, Istituto Nanoscienze-CNR and Scuola Normale Superiore, Pisa I-56127, Italy \\ ${ }^{3}$ Department of Physics and Astronomy, University of Florence, Sesto Fiorentino (FI) I-50019, Italy
}

(Received 29 June 2021; accepted 26 August 2021; published 30 September 2021)

\begin{abstract}
Inspired by the idea that quantum computers can be useful in advancing basic science, we use a quantum processor to experimentally validate a number of theoretical results in non-equilibrium quantum thermodynamics, that were not (or were very little) corroborated so far. In order to do so, we first put forward a novel method to implement the so called two-point measurement scheme, which is at the basis of the study of nonequilibrium energetic exchanges in quantum systems. Like previously established methods, our method uses an ancillary system, but at variance with them, it provides direct access to the energy exchange statistics, and is, accordingly more effective, at least when applied to small quantum systems. Using a quantum computer as a remotely programmable experimental platform, we first validate our ancilla-assisted two-point measurement scheme, and then apply it to (i) experimentally verify that fluctuation theorems are robust against projective measurements, a theoretical prediction, which was not validated so far; (ii) experimentally verify the so-called heat-engine fluctuation relation, by implementing a SWAP quantum heat engine; (iii) experimentally verify that the heat-engine fluctuation relation holds for measurement-fueled quantum heat engines, by implementing the design at the basis of the so-called quantum-measurement-cooling concept. For both engines, we report the measured average heat and work exchanged and single out their operation mode. Our experiments constitute an experimental basis for the understanding of the nonequilibrium energetics of quantum computation and for the implementation of energy-management devices on quantum processors.
\end{abstract}

DOI: 10.1103/PRXQuantum.2.030353

\section{INTRODUCTION}

With the tremendous and fast advancements of quantum technologies, quantum computers have recently become a reality. Their development proceeds fast both in terms of the increasing number of quantum logical units (the qubits) that compose the processors, and in terms of the decreasing error accompanying the quantum information processing [1]. In the effort of improving the efficacy of quantum processors, understanding and mastering the disturbing thermal effects occurring during their operation is of crucial importance. In this regard quantum computation can largely benefit from a field of investigation

\footnotetext{
*asolfane@sissa.it

†asantini@sissa.it

\$michele.campisi@nano.cnr.it
}

Published by the American Physical Society under the terms of the Creative Commons Attribution 4.0 International license. Further distribution of this work must maintain attribution to the author(s) and the published article's title, journal citation, and DOI. that has become recently known as quantum thermodynamics [2-4]. Quantum thermodynamics is concerned with all the thermodynamic phenomena that may occur at the quantum level, ranging from thermal transport, to the fluctuations of thermodynamic quantities (notably heat and work), and in the way those can be mastered, e.g., by realizing nanoscale quantum heat engines and refrigerators.

Most notably, not only can quantum computing benefit from quantum thermodynamics, but also the latter can benefit from the former: A number of theoretical results in quantum thermodynamics, that can be experimentally checked with current quantum processors, are in fact still little if not at all corroborated by experimental evidence. Among them are the fluctuation relation for arbitrary open quantum systems [5], the robustness of fluctuation relations against perturbation induced by projective measurements [6,7], the fluctuation relation for heat engines [8,9], the realization of two-qubit and two-stroke engines, e.g., the SWAP engine [10], and the so-called quantum-measurement cooling [11], whereby a refrigeration mechanisms is set by the very action of quantum measurement, without the aid of any feedback mechanism. 
With this work we substantially fill that gap by reporting the results of a number of new experiments that we perform on IBM quantum processors [12].

All our experiments are based on the so-called twopoint measurement (TPM) scheme [13-17] whereby the energy of the quantum system of interest is measured before and after an interaction with other agents (e.g., an external work source, another quantum system, a thermal environment or a measuring apparatus) has taken place. One problem that needs to be faced when implementing such a scheme is that often projective measurements are so invasive that they destroy the possibility to continue the experiment after the outcome of the measurement has been produced. One way to circumvent this problem is to implement a Ramsey-like interferometric scheme where the information on the statistics of energy changes that would be obtained by subtracting the final and initial measurement outcome is encoded in the state of an ancillary qubit $[18,19]$ that is probed only at the end of the protocol. One drawback of this method is that it actually measures the characteristic function $G(u)$, namely the Fourier transform of the wanted statistics, $\mathcal{P}(W)$. That function needs to be sampled at a great number of points $u$, in order for $\mathcal{P}(W)$ to be efficiently extracted, and a great number of runs of the same experimental protocol must be repeated in order to collect sufficient statistics to achieve a good estimation of the characteristic function at each specific value $u$ of its argument. The method has been demonstrated in Ref. [20]. Another method has been proposed by Roncaglia et al. [21]. In this method an ancillary system, appropriately coupled to the system of interest is used as a pointer whose position is proportional to the energy of the system. Looking at the pointer overall displacement accompanying the system evolution, provides access to the work distribution. This method has been experimentally demonstrated in a Stern-Gerlachtype experiment with $\mathrm{Rb}$ atoms on an atom chip [22]. Its implementation in a quantum computer would require two quantum Fourier transform (QFT) gates to obtain a coarse-grained version of the work distribution, the larger the Hilbert space of the ancilla, the finer the resolution of the distribution [21]. Yet another method to implement the TPM scheme has been employed in Refs. [23,24]. In those experiments a sequence of state-sensitive fluorescence detections is applied on a trapped ion, until it gets into the vibrational ground state. Counting the number $n$ of steps in the sequence tells what was the initial state of the ion, which can then be recreated by applying the inverse sequence of $n$ excitation steps. This engineered projective measurement might not be easily portable on other quantum platforms.

Another problem that one typically faces when experimentally addressing quantum thermodynamics results is that normally they are based on the assumption that the quantum systems that are being manipulated and measured are initially in an equilibrium thermal state, while quantum technologies, including quantum processors, typically allow for the initialization of quantum systems in a specific pure state [25]. This problem can be circumvented by randomly initializing the system in a certain energy eigenstate with the according Gibbs probability, thus emulating, rather than creating, a genuine thermal state, as, e.g., in Refs. [26,27], and has the advantage of being immune from the initial measurement issue mentioned above [28]. However, a number of quantum algorithms, possibly involving a number of auxiliary qubits, have been devised to prepare a Gibbs state on quantum processors [25,29-34], some of which are based on the creation of the so-called thermofield double (TFD) state [31,32]. Finally, another possibility is to prepare the system in a coherent superposition of energy eigenstates $|n\rangle$ with complex coefficients $c_{n}$ being the square roots of the Gibbs weights. Letting the system decohere for a sufficiently long time, brings the system to the wanted thermal state $[23,24]$.

Here we show that using the TFD state technique to prepare the system in a thermal state, allows the TPM statistics $\mathcal{P}(W)$ to be exactly recovered in a most direct way, without incurring the difficulties associated to the TPM scheme, thus solving the two problems mentioned above at once.

We implement our method, which we dub the ancillaassisted two-point-measurement (AATPM) scheme on IBM quantum processors, and illustrate its employment for experimentally studying a number of problems in nonequilibrium quantum thermodynamics. We remark that IBM has recently added the possibility to insert projective measurement within a quantum circuit (not only at its end), a possibility that is not yet available on other existing quantum computing platforms, which we use to validate our method.

In Sec. II we illustrate the theory at the basis of our AATPM scheme and validate its efficacy against the native IBM scheme, by using the accuracy with which the Jarzynski identity is reproduced as an experimental benchmark. In Sec. III we experimentally verify the validity of the fluctuation relation in the presence of intermediate projective measurements, a result that has been predicted in Ref. [6,7]. In Sec. IV A we report the implementation of the two-stroke and two-qubit SWAP engine [10] design and use it to verify the quantum heat-engine fluctuation relation predicted in Ref. [8]. We characterize the mode of operation of the device by measuring the actual energy exchanges occurring in the engine. In Sec. IV B we report on the implementation of the heat-engine design at the basis of the so-called quantum-measurement cooling [11]. We experimentally validate the prediction that the heatengine fluctuation relation should be obeyed in this case as 
well, and characterize the mode of operation of the device as we did for the SWAP engine design.

\section{ANCILLA-ASSISTED TWO-POINT MEASUREMENT: THEORY AND EXPERIMENTAL VALIDATION}

\section{A. Theory}

We start by considering the prototypical nonequilibrium quantum thermodynamics scenario of a quantum system prepared in a thermal state at some inverse temperature $\beta$ and being subjected to a measurement-drivingmeasurement (MDM) protocol, where the measurement stages are projective measurements of the system Hamiltonian, and the driving stage is the application of some external forcing that induces some unitary evolution $U$ $[16,17]$. The extension to more complex situations where, for example, the system is multipartite and each part is initialized at its own initial inverse temperature $\beta_{i}$, or the evolution is not unitary (e.g., a unitary interrupted by projective measurements) is straightforward.

We are interested in the statistics $\mathcal{P}(W)$ of the energy change of our quantum system (i.e., the work $W$ ), as recorded in a single realization of the MDM protocol. It reads $[16,17]$

$$
\mathcal{P}(W)=\sum_{m, n} p_{n} p_{m \mid n} \delta\left[W-\left(E_{m}-E_{n}\right)\right],
$$

where $p_{n}=e^{-\beta E_{n}} / Z$ is the probability to find the system at energy $E_{n}$ in the first measurement, and $p_{m \mid n}$ is the probability of finding the system at energy $E_{m}$ in the second measurement given that it was found at $E_{n}$ in the first measurement. Its expression in terms of $U$ and the system Hamiltonian eigenprojectors $\Pi_{k}^{s}$ (such that $H \Pi_{k}^{s}=E_{k} \Pi_{k}^{s}$ ) reads

$$
p_{m \mid n}=\operatorname{Tr}_{S} \Pi_{m}^{s} U \Pi_{n}^{s} U^{\dagger} \Pi_{m}^{s},
$$

where $\operatorname{Tr}_{S}$ denotes the trace operation in the system Hilbert space.

Our approach to experimentally obtain the joint probability $p_{m n}=p_{m \mid n} p_{n}$ consists in first imprinting the information about the initial state of our system in an ancillary quantum system (which is a copy of the system itself), let then the system evolve under $U$, and finally measure both ancilla and system energies, so as to obtain, respectively, $E_{n}$ and $E_{m}$.

The information-imprinting stage is achieved by creating the entangled state:

$$
|\psi\rangle=\sum_{n} c_{n}|n\rangle_{s} \otimes|n\rangle_{a} .
$$

In practice the state $|\psi\rangle$ can be created by preparing the system and ancilla in some factorized state $\left|\psi_{0}\right\rangle_{s} \otimes\left|\psi_{0}\right\rangle_{a}$ and then applying an appropriate entangling unitary $V$. Quite remarkably, by designing the unitary $V$ in such a way that $c_{n}=\sqrt{p_{n}}$ one can so simultaneously achieve the task of physically preparing the wanted mixed state for the system:

$$
\rho_{S}=\operatorname{Tr}_{A}|\psi\rangle\langle\psi|=\sum_{n} p_{n} \Pi_{n}^{s}
$$

with the populations $p_{n}=e^{-\beta E_{n}} / Z$ relative to the system energy eigenstates $|n\rangle_{s}$ (here $\operatorname{Tr}_{A}$ denotes trace over the ancilla Hilbert space). When $c_{n}=\sqrt{e^{-\beta E_{n}} / Z}$, the state in Eq. (3) is the so-called thermofield double state $[31,32]$.

With the preparation in Eq. (3) the joint probability $Q_{m n}$ of measuring $E_{n}$ in the ancilla and $E_{m}$ in the system equals the joint probability $p_{m n}=p_{n} p_{m \mid n}$, Eq. (2):

$$
\begin{aligned}
Q_{m n} & =\operatorname{Tr}\left(\Pi_{m}^{s} U \otimes \Pi_{n}^{a}\right)|\psi\rangle\langle\psi|\left(U^{\dagger} \Pi_{m}^{s} \otimes \Pi_{n}^{a}\right), \\
& =p_{n} \operatorname{Tr}\left(\Pi_{m}^{s} U \otimes \mathbb{1}_{a}\right)\left(\Pi_{n}^{s} \otimes \Pi_{n}^{a}\right)\left(U^{\dagger} \Pi_{m}^{s} \otimes \mathbb{1}_{a}\right), \\
& =p_{n} \operatorname{Tr}_{S} \Pi_{m}^{s} U \Pi_{n}^{s} U^{\dagger} \Pi_{m}^{s}=p_{m \mid n} p_{n}=p_{m n} .
\end{aligned}
$$

Here $\Pi_{n}^{a}$ are the ancilla eigenprojectors, and $\mathbb{1}_{a}$ denotes the identity operator in the ancilla Hilbert space. In going from the first to the second line we use the salient equation

$$
\left(\mathbb{1}_{s} \otimes \Pi_{n}^{a}\right)|\psi\rangle=\sqrt{p_{n}}|n\rangle_{s} \otimes|n\rangle_{a},
$$

which reflects the essential feature of entanglement. In going from the second to the third line we use the rule $\operatorname{Tr}=\operatorname{Tr}_{A} \operatorname{Tr}_{S}$ and assume the projector operators have unit trace, specifically $\operatorname{Tr}_{A} \Pi_{n}^{a}=1$.

Note that, as can be seen from the above derivation, since the measurement operators of the ancilla $\mathbb{1}_{s} \otimes \Pi_{n}^{a}$ commute with the operators $\Pi_{m}^{s} U_{s} \otimes \mathbb{1}_{a}$, whether they are performed before, after or simultaneously with the final system measurement is irrelevant. The information on the initial state of the system is encoded in the ancilla once and for all, and can be retrieved later at any time, without influencing the system.

We remark that the method does not rely on the fact that the evolution of the main qubit is unitary, hence our derivation continues to hold for a generic evolution described by a generic quantum channel. In the following we, in fact, demonstrate it both for unitary and nonunitary channels, e.g., when a qubit is subject to projective measurements, or when it interacts with other qubits.

Figure 1 shows the quantum-circuit representation of the AATPM scheme, for the case of a system being composed by a single qubit with Hamiltonian

$$
H_{q_{0}}=\frac{\omega}{2} \sigma_{z}^{0}
$$

with $\omega$ the energy-level spacing. The qubit of interest is denoted as $q_{0}$ while $q_{1}$ is the ancilla. The first block, 


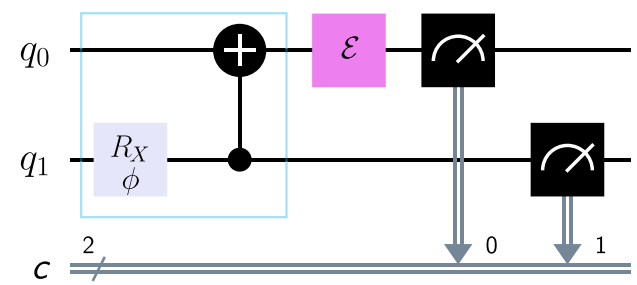

FIG. 1. Quantum-circuit representation of the AATPM scheme. The two-qubit operation in the blue box creates the purified state, Eq. (9), that both prepares the main qubit $q_{0}$ in a thermal mixture, and imprints the information about its state in the ancilla qubit $q_{1}$. The main qubit evolves according to a generic map $\mathcal{E}$, pink box. Finally, both qubits are read, providing information about the state of the main qubit before and after the evolution.

consisting of a rotation of an angle

$$
\phi=2 \arctan \left(e^{\beta \omega / 2}\right),
$$

along the $X$ direction of the qubit $q_{1}$ followed by a CNOT gate prepares the entangled state:

$$
\begin{aligned}
|\psi\rangle= & \frac{1}{\sqrt{2 \cosh (\beta \omega / 2)}}\left(e^{-\beta \omega / 4}|0\rangle_{0} \otimes|0\rangle_{1}\right. \\
& \left.+e^{\beta \omega / 4}|1\rangle_{0} \otimes|1\rangle_{1}\right) .
\end{aligned}
$$

Here $|k\rangle_{i}$ denotes the energy eigenstates of the system $(i=0)$, and ancilla $(i=1)$. Accordingly, the qubit of interest is prepared in the Gibbs state

$$
\rho_{q_{0}}=\operatorname{Tr}_{q_{0}}|\psi\rangle\langle\psi|=\frac{e^{-\beta \omega \sigma_{z}^{0} / 2}}{2 \cosh (\beta \omega / 2)},
$$

of rescaled inverse temperature

$$
\beta \omega=2 \ln \tan (\phi / 2) .
$$

Subsequently it evolves according to a generic evolution $\mathcal{E}$, and finally both qubits are read in their respective $\sigma_{z}$ basis. By repeating the circuit $\mathcal{N}$ times one can experimentally determine the probabilities $p_{q_{i}}^{k}$ to find qubit $q_{i}$ in the eigenstate $|k\rangle_{i}$, and hence measure the joint probability $p_{m n}$ as $p_{m n}=Q_{m n}=p_{q_{0}}^{m} p_{q_{1}}^{n}$.

\section{B. Experimental validation}

It is well known that quantum systems prepared in a thermal state and then evolving under a unitary driving, satisfy the celebrated Jarzynski identity

$$
\left\langle e^{-\beta W}\right\rangle=e^{-\beta \Delta F},
$$

where $\beta$ is the initial inverse temperature, $W$ is the work exchanged during the driving protocol, the average $\langle\cdots\rangle$ is taken with respect to the work statistics defined in Eq. (1), while $-\Delta F$ is the difference between the initial free energy and the free energy the system would have if it was in equilibrium, at inverse temperature $\beta$, at the end of the protocol.

In order to validate our AATPM method we implement it on IBM quantum processors and use it to study the validity of the Jarzynski equality. In our implementation, after preparation in the thermal state, the qubit of interest $q_{0}$ is evolved according to a Hadamard gate [35], which in the computational basis $\sigma_{z}$ reads

$$
U=\frac{1}{\sqrt{2}}\left(\begin{array}{cc}
1 & 1 \\
1 & -1
\end{array}\right)
$$

with a $\Delta F=0$. We collect the qubits statistics over a sample of size $\mathcal{N}=8192$, and repeat the experiments for various values of the angle $\phi$.

The results are benchmarked against the results obtained by directly implementing the TPM scheme using the newly added IBM capabilities.

Figure 2(a) shows the measured values of the probabilities $P_{+} \doteq Q_{01}, P_{-} \doteq Q_{10}$, and $P_{0} \doteq Q_{00}+Q_{11}$, that the qubit undergoes, respectively, the energy changes $\omega,-\omega, 0$, as a function of the rescaled inverse temperature $\beta \omega=2 \ln \tan (\phi / 2)$, Eq. (11). The error bars in Fig. 2 represent the statistical error due to the finiteness of our samples, and are accordingly calculated as $\delta Q_{01}=\delta Q_{10} \simeq$ $1 / \sqrt{\mathcal{N}}$ and $\delta\left(Q_{00}+Q_{11}\right) \simeq 2 / \sqrt{\mathcal{N}}$.

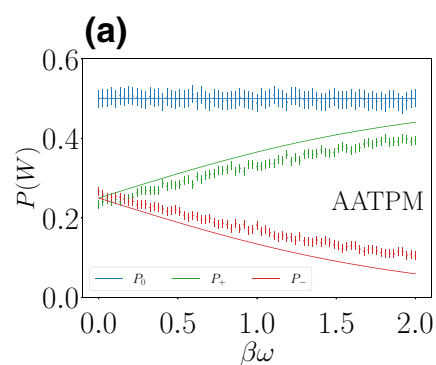

(c)

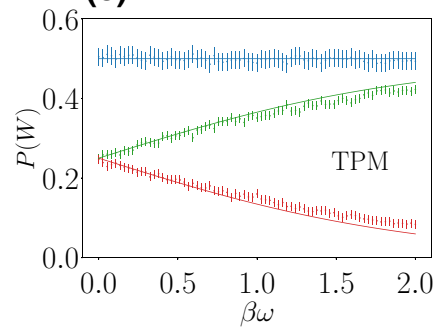

(b)

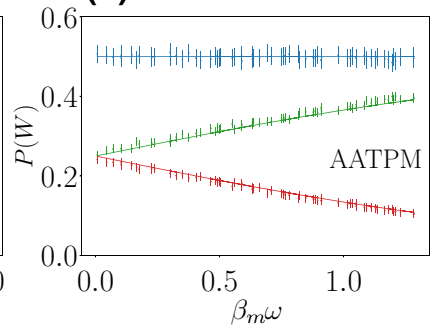

(d)

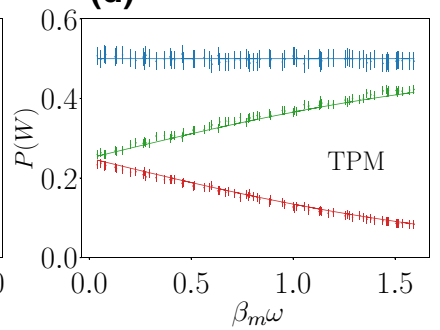

FIG. 2. (a),(c) Work statistics as a function of nominal rescaled inverse temperature for the standard TPM scheme and the AATPM scheme, respectively, for a qubit driven by a Hadamard gate. (b),(d) Same as (a),(c) but as a function of measured rescaled inverse temperature. Dots, experimental data; solid lines, theory. 
Figure 2(c) shows the same quantities as Fig. 2(a) but for the standard TPM scheme, i.e., obtained by replacing the measurement on the ancilla, with a direct measurement on the qubit of interest, before the application of the driving (Hadamard gate).

Figures 2(a) and 2(c) evidence a systematic discrepancy between observed and theoretical data, for both methods. This is due to a mismatch between the nominal rescaled inverse temperature $\beta \omega$, Eq. (11) and the actual rescaled inverse temperature acquired by the qubits after the TFD state preparation. In the AATPM scheme the latter can be read off the measured populations of the ancilla as

$$
\beta_{m} \omega=\ln \left[p_{q_{1}}^{1} / p_{q_{1}}^{0}\right]
$$

Similarly, in the standard TPM scheme, they are read off the populations of the first measurement of the qubit of interest.

Figures 2(b) and 2(d) report the measured probabilities $P_{ \pm}, P_{0}$ as a function of the measured rescaled inverse temperature $\beta_{m} \omega$. Excellent agreement with the theoretical expectations is now achieved for both methods, which perform equally well. This demonstrates the validity and efficacy of the AATPM scheme.

In Fig. 3 we plot the quantity $\left\langle e^{-\beta_{m} W}\right\rangle$ as a function of $\beta_{m} \omega$ for the two methods, blue triangles refer to the AATPM scheme while the red crosses are obtained with the standard TPM scheme. As in Fig. 2, the statistics is generated for each value of $\beta_{m} \omega$ from a sample of size $\mathcal{N}=$ 8192. In order to estimate the statistical uncertainty on our estimation of the above quantity we select a few values of temperature, repeat the estimation of $\left\langle e^{-\beta_{m} W}\right\rangle k=225$ times, and take the standard deviation, $\sigma$, of the obtained values as a measure of the statistical error affecting our data. Such a value of statistical error is reported for 4 values of $\beta_{m} \omega$ in the inset of Fig. 3. Note how the error increases (approximately) linearly with increasing $\beta_{m} \omega$. We thus linearly interpolated the data in order to obtain an estimate of the statistical error for all values of $\beta_{m} \omega$. The two colored shadows in Fig. 3, are the regions contained within $3 \sigma$ of the observed value, for both methods. Excellent agreement with Eq. (12) is found for all values of $\beta_{m} \omega$. The observed fact that the quantity $\left\langle e^{-\beta_{m} W}\right\rangle$ tends to be underestimated with increasing $\beta_{m} \omega$, while the error increases, has a genuinely statistical origin, associated to the nonlinear character of the exponential.

\section{ROBUSTNESS OF FLUCTUATION THEOREMS TO INTERMEDIATE PROJECTIVE MEASUREMENTS}

With Refs. [6,7] it has been established that the Jarzynski equality, Eq. (12), continues to hold true even if, during the driving protocol, an arbitrary number of projective measurements of observables, which possibly do not commute with the system Hamiltonian are performed. Here we

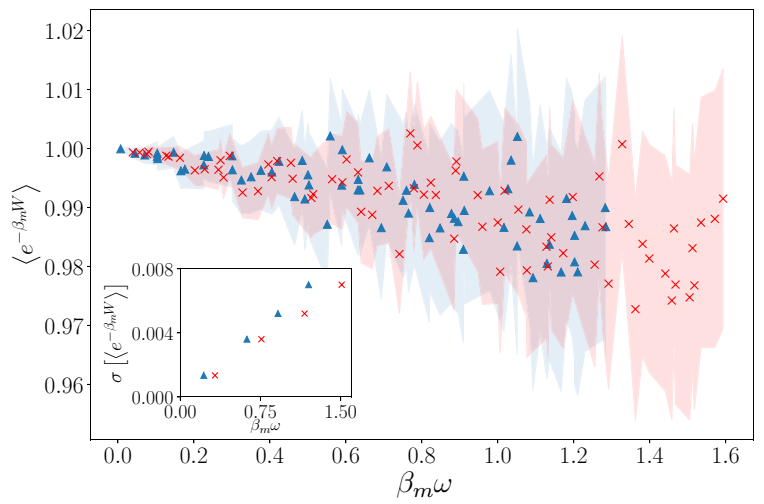

FIG. 3. Average exponentiated work $\left\langle e^{-\beta_{m} W}\right\rangle$ for a qubit driven by a Hadamard gate, as measured using the standard TPM (red crosses) and the AATPM (blue triangles), for various values of measured rescaled inverse temperature $\beta_{m} \omega$. Inset: the relative statistical uncertainty for some values of $\beta_{m} \omega$ obtained as the standard deviations of averages as detailed in the text.

experimentally check the validity of that prediction, by taking advantage of the newly added possibility to perform projective measurements at any time during the execution of a quantum circuit, on IBM processors.

The quantum circuit employed to experimentally validate the robustness of the Jarzynski identity to intermediate measurements is depicted in Fig. 4. The circuit starts with the creation of the pure state, Eq. (9), according to the procedure described above.

The qubit of interest $q_{0}$ is then first measured in the computational basis $\sigma_{z}$ (that implements the first measurement of the standard TPM scheme), and then undergoes a sequence of identical unitary evolutions $U$, spaced out by projective measurements of some observable $A$. Specifically, in our experiments, we choose $U$ and $A$ as not commuting with each other:

$$
\begin{aligned}
& U=R_{Y}(\pi / N)=e^{-i \pi \sigma_{y} /(2 N)}, \\
& A=\sigma_{x} .
\end{aligned}
$$

We remark that the IBM hardware is equipped with the possibility of performing measurements in the $\sigma_{z}$ basis of each qubit, only. In order to perform a measurement in a different basis one should accordingly first apply the basis-change unitary gate $V$ that maps the wanted measurement basis onto the $\sigma_{z}$ eigenbasis, then measure along $\sigma_{z}$, and finally apply the inverse unitary gate $V^{-1}[11]$. To see that, note that if $\Pi_{k}$ are the eigenprojectors associated to the measurement basis, which is available in a certain setup, and $\pi_{k}$ are the eigenprojectors associated to the wanted measurement basis (i.e., $A=\sum_{k} a_{k} \pi_{k}$, with $a_{k}$ the eigenvalues of $A$ ), it is

$$
\pi_{k}=V^{-1} \Pi_{k} V .
$$




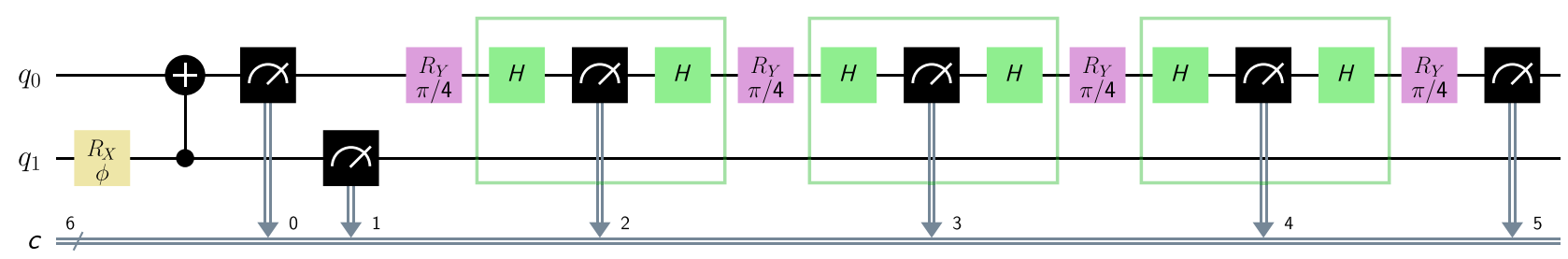

FIG. 4. Quantum circuit employed for the experimental validation of the Jarzynski equality, Eq. (12), in the presence of $N-1$ intermediate projective measurements (the case $N=4$ is displayed). The green box operations are projective measurements in the $\sigma_{x}$ basis. The main qubit $q_{0}$ initial state is both read off directly, according to the standard TPM, and indirectly by reading the state of the ancilla, according to the AATPM scheme. The outcome of the intermediate measurements is ignored.

Specifically, the $\sigma_{x}$ measurement is implemented in the present architecture, by sandwiching the $\sigma_{z}$ measurement between two Hadamard gates (see green gates in Fig. 4).

After the train of intermediate projective measurements both the qubit of interest and ancilla are measured in their respective $\sigma_{z}$ basis to collect the AATPM probabilities $p_{q_{i}}^{k}$ as in the previous section.

As discussed in Refs. [6,7], the conditional probability $p_{m \mid n}$ that qubit $q_{0}$ is found in state $|m\rangle$ at the last measurement, provided that it was found in state $|n\rangle$ in the first measurement, reads

$$
p_{m \mid n}=\sum_{a_{1}} \cdots \sum_{a_{N-1}} p_{m \mid a_{N-1}} \prod_{k=1}^{N-2} p_{a_{k+1} \mid a_{k}} p_{a_{1} \mid n}
$$

where the $p_{a_{k+1} \mid a_{k}}=\left|\left\langle a_{k+1}|U| a_{k}\right\rangle\right|^{2}$ are the condition probabilities that the output of the $k+1$ th measurement is $a_{k+1}$, given that the output of the $k$ th measurement is $a_{k}$, while $p_{m \mid a_{N}}=\left|\left\langle m|U| a_{N}\right\rangle\right|^{2}$ and $p_{a_{1} \mid n}=\left|\left\langle a_{1}|U| n\right\rangle\right|^{2}$.

With our choice of $U$ and $A$, the transition probabilities $p_{m \mid n}$, read, for $N>1$,

$$
\begin{aligned}
& p_{0 \mid 0}=p_{1 \mid 1}=\frac{1}{2}\left[1-\cos ^{N-2}\left(\frac{\pi}{N}\right)+\cos ^{N}\left(\frac{\pi}{N}\right)\right],(19 \mathrm{a}) \\
& p_{0 \mid 1}=p_{1 \mid 0}=\frac{\sin ^{2}(\pi / 2 N)\left[1+\cos ^{N-2}(\pi / N) \sin ^{2}(\pi / N)\right]}{[1-\cos (\pi / N)]} .
\end{aligned}
$$

For $N=1$, they read $p_{0 \mid 0}=p_{1 \mid 1}=0, p_{0 \mid 1}=p_{1 \mid 0}=1$. These analytical expressions are used to compute the values of $P_{+}, P_{-}, P_{0}$ entering the statistics of energy change of the qubit, as in the previous section. Those are plotted for different values of $N$ and fixed nominal inverse rescaled temperature $\beta \omega=1$, in Fig. 5(a), and compared with the values obtained from our experiments. Sample size and errors are evaluated as in the previous section.

Figure 5(b) shows the quantity $\left\langle e^{-\beta_{m} \Delta E}\right\rangle$ and its statistical uncertainty (shown in the inset for a few points), estimated as in the previous section. Note that we now denote the energy change of the qubit as $\Delta E$, rather than $W$, because now the qubit not only exchanges energy in the form of work (during the unitary gates) but also in the form of so-called "quantum heat" (during the measurements) [36]. We observe that there is a very good agreement between the ancilla-assisted results, blue triangles, and those obtained with the standard two-point measurement scheme, red crosses. Most importantly, we observe that the relative deviation of the experimental data and that theoretical prediction, (i.e., the value 1) is less than $4 \%$, for all values of $N$, thus experimentally verifying the
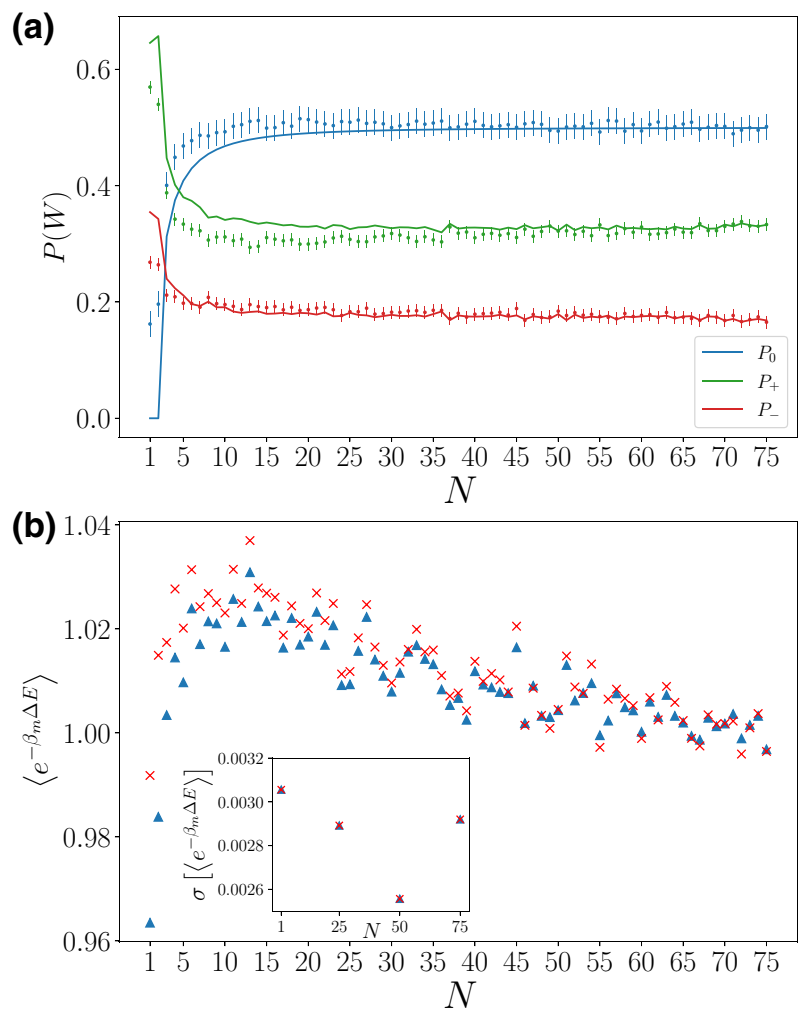

FIG. 5. (a) Work statistics with $N-1$ intermediate projective measurements. (b) Robustness of the Jarzynski identity in the presence of projective measurements. The inset shows the statistical uncertainty for a few values of $N: N=1,25,50,75$. The blue triangles refer to the ancilla-assisted method while the red crosses are obtained with the standard two-point measurement scheme. 
robustness of the fluctuation relation to intermediate measurements. We remark that our estimated error, see inset of Fig. 5(b), accounts only for the statistical errors.

We notice that a better agreement is found for larger number of intermediate measurements, for both plots in Fig. 5. In our understanding this fact has a twofold origin. On one hand, in the limit of large $N$ it is, in our case, see Eq. (19)

$$
\lim _{N \rightarrow \infty} p_{m \mid n}=\frac{1}{2}, \quad \forall m, n
$$

Namely, as a consequence of the protocol applied on the qubit, the latter gets randomized into the completely mixed state $\rho=\mathbb{1} / 2$. The phenomenon of randomization induced by a train of measurements has been observed and discussed earlier, see e.g., Refs. [7,37-39], but here it occurs as a consequence a different mechanism. After preparation in a state that is diagonal in the $\sigma_{z}$ basis, the qubit undergoes a rotation of a vanishingly small angle in the large $N$ limit. That is in the limit of infinite $N$ it remains along $z$, and the subsequent measurement in the $\sigma_{x}$ basis fully randomize it, leaving in the fully mixed state $\mathbb{1} / 2$. The subsequent evolution interrupted by further measurements in the same basis of $\sigma_{x}$, is frozen due to the quantum Zeno effect [40,41], i.e., the system does not evolve at all, thus remaining at all times in the fully mixed state $\mathbb{1} / 2$. As a consequence all correlations between initial and final energy measurements are lost and the joint probability factorizes $p_{m n}=p_{n} p_{m}=p_{n} / 2$. Accordingly, the average exponentiated energy change is not subject to the sources of error that typically affect the conditional probability $p_{m \mid n}$ :

$\left\langle e^{-\beta \Delta E}\right\rangle=\sum_{m} \frac{e^{-\beta E_{m}}}{2} \sum_{n} p_{n} e^{\beta E_{n}}=\frac{Z}{2} \sum_{n} \frac{e^{-\beta E_{n}}}{Z} e^{\beta E_{n}}=1$.

On the other hand, we notice that rotations of angles close to $\pi$ are affected by larger error, see Fig. 6. It shows the measured values of $\left\langle\sigma_{z}\right\rangle$ as a function of the rotation angle $\alpha$ against its theoretical expectation, for a single qubit that is initialized in $|0\rangle$ and is rotated according to the gate $R_{Y}(\alpha)$, for different values of $\alpha$. The figure evidences that larger deviations are found for angles close to $\pi$. In our experiment, the lower is $N$, the closer are rotation angles to $\pi$, hence the larger is the error brought up by each single rotation. This could be another reason why better agreement is observed for large $N$.

\section{HEAT-ENGINE FLUCTUATION RELATION}

Any driven bipartite system subject to a cyclic driving and evolving from the tensor product of Gibbs states for

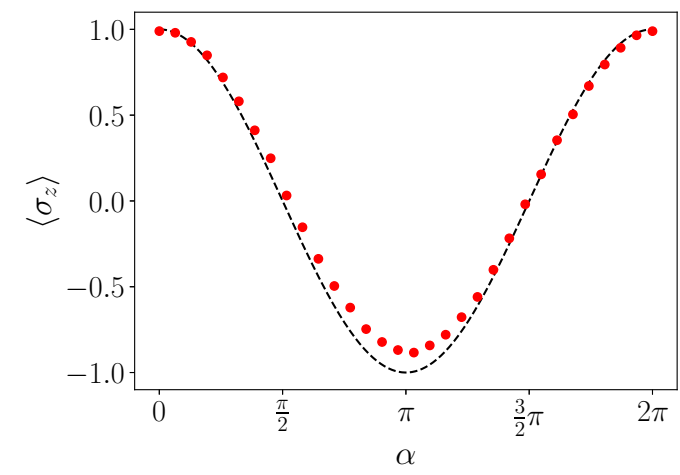

FIG. 6. Expectation value of $\sigma_{z}$ for a single qubit initialized in $|0\rangle$ after the application of a $R_{Y}(\alpha)$ gate. Red dots, experimental result; dashed line, theoretical prediction.

each subsystem obeys the following multivariate fluctuation relation $[10,16,17,42]$ :

$$
\left\langle e^{-\beta_{1} \Delta E_{1}-\beta_{2} \Delta E_{2}}\right\rangle=1 .
$$

When the work $W$, i.e., the sum of energy changes $\Delta E_{1}+$ $\Delta E_{2}$ of the two parts of the system, is not negligible, as in the case of heat engines, the above is often referred to as the heat-engine fluctuation relation $[8,10]$. The average $\langle\cdots\rangle$ is now over the joint statistics of the energy exchanges $\Delta E_{1}$ and $\Delta E_{2}$, reading

$$
\begin{aligned}
P\left[\Delta E_{1}, \Delta E_{2}\right]= & \sum_{n_{1}, n_{2}, m_{1}, m_{2}} p_{n_{1} n_{2}} p_{m_{1} m_{2} \mid n_{1} n_{2}} \\
& \times \delta\left[\Delta E_{1}-\left(E_{m_{1}}^{1}-E_{n_{1}}^{1}\right)\right] \\
& \times \delta\left[\Delta E_{2}-\left(E_{m_{2}}^{2}-E_{n_{2}}^{2}\right)\right],
\end{aligned}
$$

where $E_{n_{i}}^{i}$ are the subsystem $i$ eigenenergies,

$$
p_{n_{1} n_{2}}=\frac{e^{-\left(\beta_{1} E_{n_{1}}^{1}+\beta_{2} E_{n_{2}}^{2}\right)}}{Z_{1} Z_{2}}
$$

are the initial populations of the system, and

$$
p_{m_{1} m_{2} \mid n_{1} n_{2}}=\left|\left\langle m_{1} m_{2}|U| n_{1} n_{2}\right\rangle\right|^{2},
$$

with $\left|n_{1} n_{2}\right\rangle$ denoting the eigenstates of the total Hamiltonian.

The heat-engine fluctuation relation, Eq. (22), has been experimentally investigated only very recently with a NMR platform (using a quantum Otto heat-engine design) by means of interferometric techniques that allow for the measurement of the characteristic function of the statistics $P\left[\Delta E_{1}, \Delta E_{2}\right]$ [43]. Here we further corroborate it by implementing a SWAP quantum heat engine on an IBM quantum processor. We remark that, for few qubit systems, as those studied here and in Ref. [43] the AATPM is 
extremely more effective, in terms of quantum processing time, than the interferometric method, employed in Ref. [43].

We further, for the first time, establish that the heatengine fluctuation relation continues to hold for quantum heat engines that rather than being fueled by unitary gates, are fueled by projective measurements, such as the quantum-measurement cooling device reported in Ref. [26].

\section{A. SWAP quantum heat engine}

The SWAP quantum heat engine, first introduced in Refs. [44-46] is a two-qubit and two-stroke heat-engine design that has recently become more and more popular as a prototype of a quantum heat engine, see e.g., Refs. [10,47,48]. Despite its broad interest, its experimental implementation has not been reported so far. Using the methods and tools developed above, we implement it on the IBM architecture. The working substance of a SWAP quantum heat engine is made of two qubits. Let

$$
H_{q_{i}}=\frac{\omega_{i}}{2} \sigma_{z}^{i}, \quad i=1,2,
$$

denote their Hamiltonians, where $\sigma_{z}^{i}$ denotes the $z$ Pauli sigma matrix of the $i$ th qubit. At the beginning of the cycle the qubits are decoupled and each at thermal equilibrium at inverse temperatures, $\beta_{i}, i=1,2$, respectively. Their initial state is then

$$
\rho=\frac{e^{-\beta_{1} H_{q_{1}}}}{Z_{1}} \otimes \frac{e^{-\beta_{2} H_{q_{2}}}}{Z_{2}}
$$

with $Z_{i}=\operatorname{Tr}_{i} e^{-\beta_{i} H_{q_{i}}}$. In the first step of the cycle, the two qubits undergo a unitary evolution according to the SWAP gate

$$
U_{\text {SWAP }}=\left(\begin{array}{llll}
1 & 0 & 0 & 0 \\
0 & 0 & 1 & 0 \\
0 & 1 & 0 & 0 \\
0 & 0 & 0 & 1
\end{array}\right)
$$

In the second step, the qubits interact each with a thermal bath at inverse temperature $\beta_{i}$, until they thermalize and the initial state $\rho$ is re-established, thus closing the cycle.

Figure 7 shows how we implement the SWAP engine on the IBM architecture. Qubits $q_{1}$ and $q_{2}$ constitute the working substance, while qubits $q_{0}$ and $q_{3}$ are their respective ancillas, which we employ to prepare them in thermal state according to Eq. (27) and to provide the AATPM capability, as described above. We then apply the SWAP gate on the main qubits $\left(q_{1}, q_{2}\right)$, and finally measure all qubits in their respective $\sigma_{z}$ basis. One could now apply appropriate gates (depending on the outcomes of the measurements) that re-establish the initial state and so close the cycle.

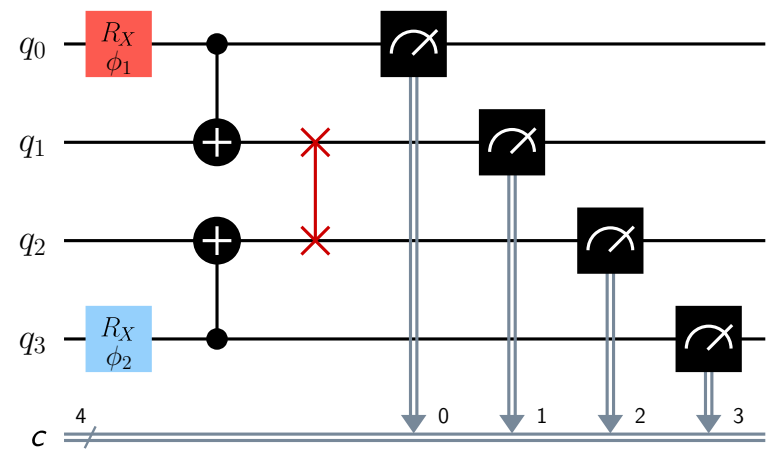

FIG. 7. Quantum-circuit implementation of the SWAP quantum heat engine. The main qubits $q_{1}, q_{2}$ are first prepared in thermal states at different temperatures with the aid of their ancillas $q_{0}, q_{3}$ and then undergo a SWAP evolution. Finally, their initial states are read off the ancillas, and their final states are read directly.

For our purposes that is not necessary, since the outcome of the measurements already contains all the information regarding the joint statistics of the energy gained by each qubit, which is sufficient for a full thermodynamic characterisation of the engine [10].

For each fixed value of the rotation angles $\phi_{1}, \phi_{2}$, we run the circuit in Fig. $7 \mathcal{N}=8192$ times, and so collect the probabilities $p_{q_{j}}^{k}$ of finding the qubit $j$ in the state $|k\rangle_{j}$ with $j=0,1,2,3$ and $k=0,1$. We recall that $p_{q_{0}}^{k}, p_{q_{1}}^{k}$ represent the probability that qubit 1 is in state $|k\rangle_{1}$ at initial time and final time, respectively. Similarly $p_{q_{3}}^{k}, p_{q_{2}}^{k}$, represent the probability that qubit 2 is in state $|k\rangle_{2}$ at initial time and final time, respectively. Extending the argument presented in Sec. II, we obtain the joint probability $p_{m_{1}, m_{2}, n_{1}, n_{2}}=$ $p_{n_{1}, n_{2}} p_{m_{1} m_{2} \mid n_{1} n_{2}}$ as $Q_{m_{1}, m_{2}, n_{1}, n_{2}}=p_{q_{0}}^{n_{1}} p_{q_{1}}^{m_{1}} p_{q_{2}}^{m_{2}} p_{q_{3}}^{n_{2}}$.

Figure 8 shows the probabilities $P_{a b}, a, b=-, 0,+$, that the energy change of qubit $q_{1}$ is $\Delta E_{1}=a \omega_{1}$, and the energy change of qubit $q_{2}$ is $\Delta E_{2}=b \omega_{2}$. Those are obtained from the joint probabilities $p_{m_{1}, m_{2}, n_{1}, n_{2}}$ via the expression

$$
P_{a b}=\sum_{m_{1}, m_{2}, n_{1}, n_{2}} p_{m_{1}, m_{2}, n_{1}, n_{2}} \delta_{m_{1}-n_{1}, a} \delta_{m_{2}-n_{2}, b},
$$

with $\delta_{i, j}$ the Kronecker symbol. For all values of nominal rescaled inverse temperatures $\omega_{i} \beta_{i}$, we find good agreement between the experimentally measured values and the theoretical ones

$$
\begin{aligned}
P_{00} & =\frac{2 \cosh \left(\beta_{1} \omega_{1} / 2+\beta_{2} \omega_{2} / 2\right)}{Z_{1} Z_{2}}, \\
P_{-+} & =\frac{e^{-\left(\beta_{1} \omega_{1}-\beta_{2} \omega_{2}\right) / 2}}{Z_{1} Z_{2}}, \\
P_{+-} & =\frac{e^{\left(\beta_{1} \omega_{1}-\beta_{2} \omega_{2}\right) / 2}}{Z_{1} Z_{2}}, \\
P_{ \pm 0} & =P_{0 \pm}=P_{ \pm \pm}=0,
\end{aligned}
$$



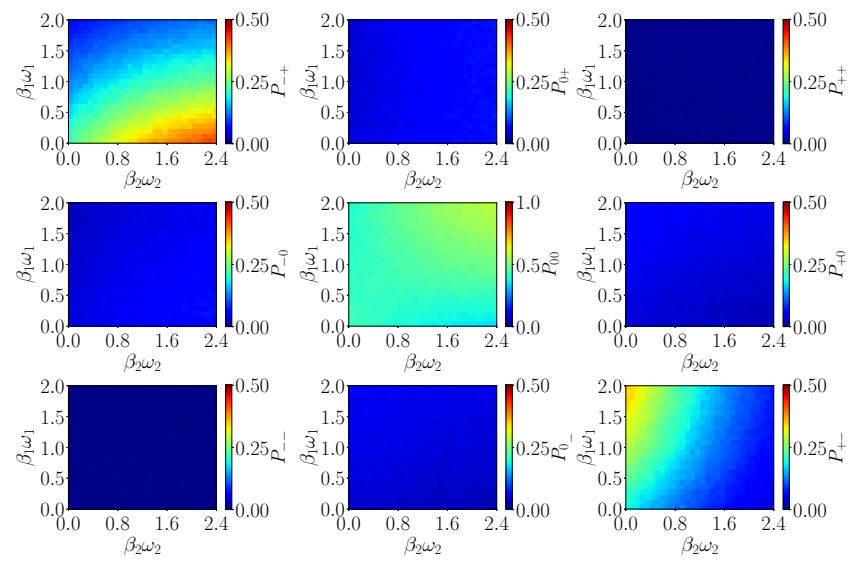

FIG. 8. Joint probabilities $P_{a b}$ with $a, b=+, 0,-$, Eq. (30), for the SWAP engine in Fig. 7, as a function of the rescaled nominal inverse temperatures $\beta_{1} \omega_{1}$ and $\beta_{2} \omega_{2}$.

where, for $\beta_{i} \omega_{i}$, we use the measured values

$$
\begin{aligned}
& \beta_{1, m} \omega_{1}=\ln \left[p_{1}^{q_{0}} / p_{0}^{q_{0}}\right], \\
& \beta_{2, m} \omega_{2}=\ln \left[p_{1}^{q_{3}} / p_{0}^{q_{3}}\right] .
\end{aligned}
$$

which, we recall, do not perfectly coincide with the nominal rescaled inverse temperatures $\beta_{i} \omega_{i}=2 \ln \tan \left(\phi_{i} / 2\right)$. The difference between theoretical values and experimental values of the $P_{a b}$ 's is always below the value 0.1 .

Figure 9 shows the quantity $\left\langle e^{-\beta_{m, 1} \Delta E_{1}-\beta_{m, 2} \Delta E_{2}}\right\rangle$ as a function of $\beta_{1} \omega_{1}, \beta_{2} \omega_{2}$. In our implementations the nominal values of the qubits frequencies are $\omega_{1} \approx 5.25 \hbar \mathrm{GHz}$ and $\omega_{2} \approx 5.17 \hbar \mathrm{GHz}$. We notice that the agreement with Eq. (22) is very good for sufficiently small values of $\beta_{i} \omega_{i}$ while the deviation from the theoretical value increases as $\beta_{i} \omega_{i}$ increase, with a trend towards the overestimation. In our understanding this is a consequence of the observed spillover of probability towards the six events labeled as $( \pm, 0),(0, \pm),( \pm, \pm)$ (see "off-diagonal" entries in the matrix of plots in Fig. 8), which should be ideally zero, see Eq. (30d).

For an ideal SWAP engine, the average energy exchanges and the average work read [10]

$$
\begin{aligned}
\left\langle\Delta E_{1}\right\rangle & =\left(\frac{1}{1+e^{\beta_{2} \omega_{2}}}-\frac{1}{1+e^{\beta_{1} \omega_{1}}}\right) \omega_{1}, \\
\left\langle\Delta E_{2}\right\rangle & =-\left(\frac{1}{1+e^{\beta_{2} \omega_{2}}}-\frac{1}{1+e^{\beta_{1} \omega_{1}}}\right) \omega_{2}, \\
W & =\left(\frac{1}{1+e^{\beta_{2} \omega_{2}}}-\frac{1}{1+e^{\beta_{1} \omega_{1}}}\right)\left(\omega_{1}-\omega_{2}\right) .
\end{aligned}
$$

Figures 10(a), 10(c), and 10(e) show the values of $\left\langle\Delta E_{1}\right\rangle / \omega_{1},\left\langle\Delta E_{2}\right\rangle / \omega_{2}$, and $\langle W\rangle / \bar{\omega}$ [where $\bar{\omega}=\left(\omega_{1}+\right.$

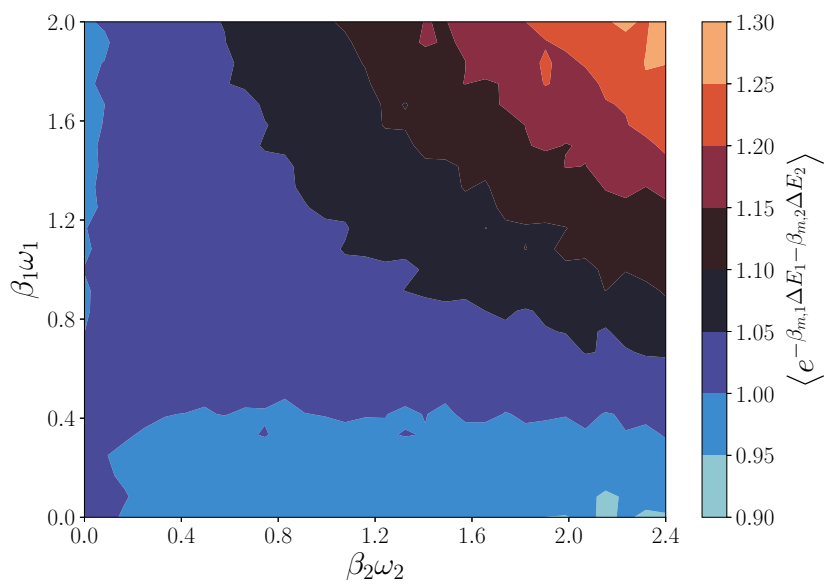

FIG. 9. Measured values of $\left\langle e^{-\beta_{m, 1} \Delta E_{1}-\beta_{m, 2} \Delta E_{2}}\right\rangle$ for the SWAP engine in Fig. 7 as a function of nominal inverse rescaled temperatures $\beta_{1} \omega_{1}, \beta_{2} \omega_{2}$.

$\left.\omega_{2}\right) / 2$ ] as obtained from the measured populations $p_{q_{j}}^{k}$ :

$$
\begin{aligned}
\left\langle\Delta E_{1}\right\rangle / \omega_{1} & =\left[\left(p_{q_{1}}^{1}-p_{q_{1}}^{0}\right)-\left(p_{q_{0}}^{1}-p_{q_{0}}^{0}\right)\right] / 2, \\
\left\langle\Delta E_{2}\right\rangle / \omega_{2} & =\left[\left(p_{q_{2}}^{1}-p_{q_{2}}^{0}\right)-\left(p_{q_{3}}^{1}-p_{q_{3}}^{0}\right)\right] / 2, \\
\langle W\rangle / \bar{\omega} & =\left(\left\langle\Delta E_{1}\right\rangle+\left\langle\Delta E_{2}\right\rangle\right) / \bar{\omega} .
\end{aligned}
$$

(a)

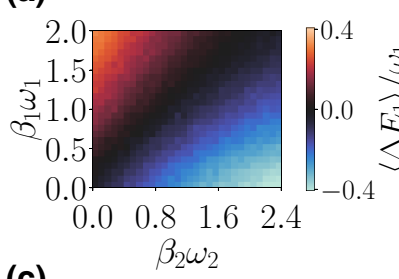

(c)

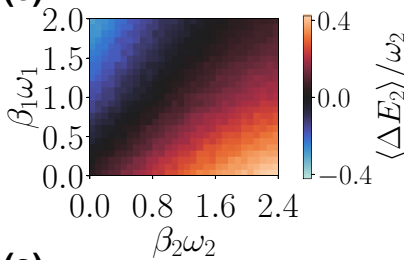

(b)

(e)

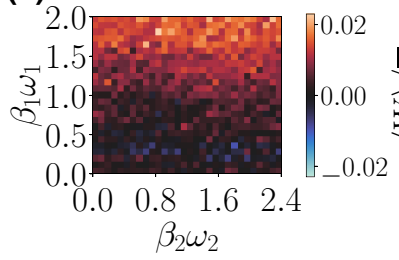

FIG. 10. (a),(c),(e) Measured rescaled energy exchanges $\left\langle\Delta E_{1}\right\rangle / \omega_{1},\left\langle\Delta E_{2}\right\rangle / \omega_{2},\langle W\rangle / \omega$, for the SWAP engine in Fig. 7 as a function of nominal inverse rescaled temperatures $\beta_{1} \omega_{1}, \beta_{2} \omega_{2}$. (b),(d),(f) Their relative experimental error. 
Figures $10(\mathrm{~b}), 10(\mathrm{~d})$, and 10(f) show their relative fluctuations estimated as

$$
\sigma\left[\left\langle\Delta E_{j}\right\rangle\right]=\frac{2 \omega_{j}}{\sqrt{\mathcal{N}}}, \quad \sigma[\langle W\rangle]=2 \frac{\omega_{1}+\omega_{2}}{\sqrt{\mathcal{N}}}
$$

with $\mathcal{N}=8192$ the size of our statistical sample.

Note that for qubits with the same resonant frequency, the expected average work is null, see Eq. (33c). In our case the two qubits' resonant frequencies are very close, and in fact the according measured work is always very small (and almost always positive), compared to the according resonant frequencies energies, see Fig. 10(e).

We recall that only four modes of operations are allowed for a generic bipartite heat engine, depending on the sign of the energy exchanges $\left\langle\Delta E_{i}\right\rangle$ and their sum, i.e., the work $\langle W\rangle$ [49]. Those are as follows: refrigerator $[R]$, i.e., the cold subsystem gets colder, the hot subsystem gets hotter while work is injected in the system; heat engine $[E]$, i.e., the cold subsystem heats up, the hot subsystem cools down while work is extracted; thermal accelerator $[A]$, i.e., work is spent to heat up the cold subsystem and cool down the hot subsystem; heater $[H]$, i.e., both subsystems receive energy from the work source.

Figure 11(a) shows the "phase diagram" of the experimentally observed modes of operation of the engine as a function of the rescaled inverse nominal temperatures of the two qubits. Figure 11(b) shows the theoretical diagram of the SWAP engine, according to the rule [10]:

$$
\begin{aligned}
& {[E]: \beta_{1} / \beta_{2} \leq \omega_{2} / \omega_{1},} \\
& {[R]: 0 \leq \omega_{2} / \omega_{1} \leq \beta_{1} / \beta_{2},} \\
& {[A]: 1 \leq \omega_{2} / \omega_{1} .}
\end{aligned}
$$

We mostly observe the thermal accelerator $[A]$ mode, and sporadically the heat engine $[E]$ and heater mode $[H]$, which accordingly cannot be considered as a robust operation (purple pixel denote cases where the error is large enough that the sign of the relevant energy change is not well defined). The refrigerator mode $[R]$ is not observed. This is because the region where we should be able to see it is too thin, see blue region in Fig. 11(b). In order to robustly implement $[R]$, it is crucial to have qubits with appreciably different level spacings, so as to widen the extension of the $[R]$ region. For example, in order to cool down a qubit, say, qubit $q_{2}$, being at temperature $1 / \beta_{2}$ it is necessary that the other qubit (that must be prepared at higher temperature $1 / \beta_{1}>1 / \beta_{2}$ ) has a larger level spacing $\omega_{1}$, see Eq. (36b).

\section{B. Quantum measurement cooling}

Quantum measurement cooling (QMC) is a cooling paradigm, recently put forward in Ref. [11], whereby a (a)

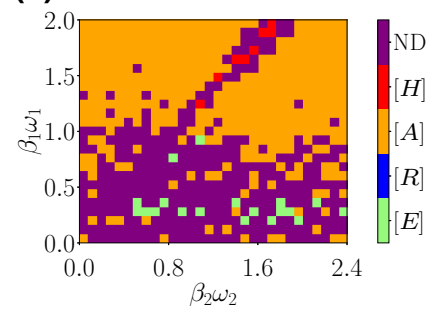

(b)

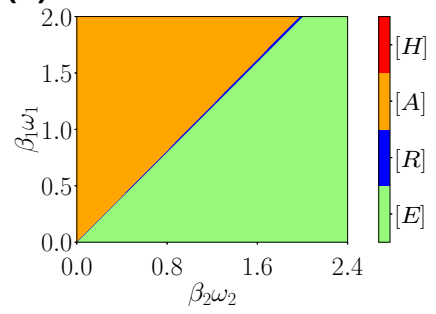

FIG. 11. (a) Experimentally observed modes of operation of the SWAP engine. (b) Theoretical prediction.

quantum refrigerator is powered by the very act of quantum measurement, by exploiting its invasiveness. As discussed in Ref. [11], such a measurement-powered cooler can be implemented by substituting, in the SWAP engine design described above, the SWAP gate with a projective measurement on a properly chosen basis. As shown in Ref. $[11,50]$ maximal cooling power (heat extracted from the cold qubit per cycle) and maximal thermodynamic cooling efficiency are achieved when the measurement basis is the singlet-triplet basis

$$
\left\{\begin{array}{l}
\left|\psi_{1}\right\rangle=|00\rangle \\
\left|\psi_{2}\right\rangle=\frac{|01\rangle+|10\rangle}{\sqrt{2}} \\
\left|\psi_{3}\right\rangle=\frac{|01\rangle-|10\rangle}{\sqrt{2}} \\
\left|\psi_{4}\right\rangle=|11\rangle
\end{array} .\right.
$$

Figure 12 shows our implementation of QMC on the IBM architecture. The quantum circuit begins with the ancillaassisted preparation of the TFD state, Eq. (27). Then a measurement on the singlet-triplet basis is performed according to the basis-change method described above, Eq. (17). In this case the basis change unitary reads

$V=\left(\begin{array}{cccc}1 & 0 & 0 & 0 \\ 0 & 1 / 2 & 1 / 2 & 0 \\ 0 & 1 / 2 & 1 / 2 & 0 \\ 0 & 0 & 0 & 1\end{array}\right)=\mathrm{CNOT} \times \mathrm{CR}_{Y}\left(-\frac{\pi}{2}\right) \times \mathrm{CNOT}$

where $\mathrm{CR}_{Y}$ is a controlled rotation along the $Y$ axis. Finally, all qubits are measured in their relative $\sigma_{z}$ basis, to give the probabilities $p_{q_{i}}^{k}$, and hence the full joint statistics of energy exchanges, as in the SWAP case.

We recall that theory predicts that the heat-engine fluctuation relation, Eq. (22), is robust to projective measurements [6], and thus should continue to hold in the present case. Figure 13 shows the quantity $\left\langle e^{-\beta_{m, 1} \Delta E_{1}-\beta_{m, 2} \Delta E_{2}}\right\rangle$ as a function of $\beta_{1} \omega_{1}$ and $\beta_{2} \omega_{2}$. We recall that the two qubits' resonant frequencies are $\omega_{1} \approx 5.25 \mathrm{GHz} \hbar$ and $\omega_{2} \approx 5.17 \mathrm{GHz} \hbar$. As in the SWAP engine setting, we 


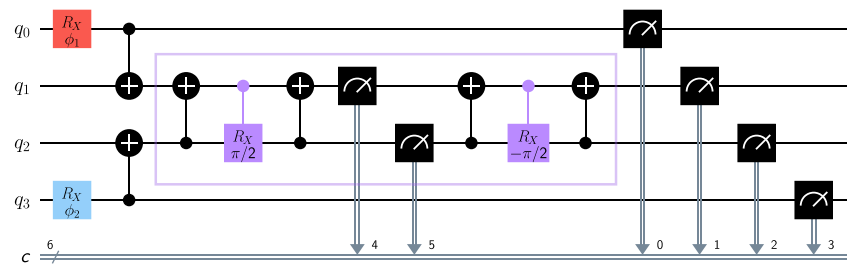

FIG. 12. Quantum-circuit implementation of the quantummeasurement-cooling heat-engine design. The main qubits $q_{1}, q_{2}$ are first prepared in thermal states at different temperatures with the aid of their ancillas $q_{0}, q_{3}$ and then undergo a measurement in the singlet-triplet basis, Eq. (37), purple box. Finally, their initial states are read off the ancillas, and their final states are read directly. The outcome of the singlet-triplet basis measurement is discarded.

observe very good agreement for small values of $\beta_{i} \omega_{i}$, and growing, but still moderate, deviations when those quantities grow. Our interpretation of this result is the same as with the SWAP engine case.

Theory predicts that, as compared to the SWAP engine, in this case all energetic exchanges are halved [11],

$$
\begin{aligned}
\left\langle\Delta E_{1}\right\rangle & =\left(\frac{1}{1+e^{\beta_{2} \omega_{2}}}-\frac{1}{1+e^{\beta_{1} \omega_{1}}}\right) \frac{\omega_{1}}{2}, \\
\left\langle\Delta E_{2}\right\rangle & =-\left(\frac{1}{1+e^{\beta_{2} \omega_{2}}}-\frac{1}{1+e^{\beta_{1} \omega_{1}}}\right) \frac{\omega_{2}}{2}, \\
W & =\left(\frac{1}{1+e^{\beta_{2} \omega_{2}}}-\frac{1}{1+e^{\beta_{1} \omega_{1}}}\right) \frac{\omega_{1}-\omega_{2}}{2} .
\end{aligned}
$$

Accordingly the "phase diagram" is identical to that of a SWAP engine.

Figures 14(a), 14(c), and 14(e) show the experimental values of $\left\langle\Delta E_{1}\right\rangle / \omega_{1},\left\langle\Delta E_{2}\right\rangle / \omega_{2}$ and $\langle W\rangle / \bar{\omega}$. Figures 14(b), $14(\mathrm{~d})$, and 14(f) show their respective relative uncertainty, computed as in Eq. (35). Note that the measured values

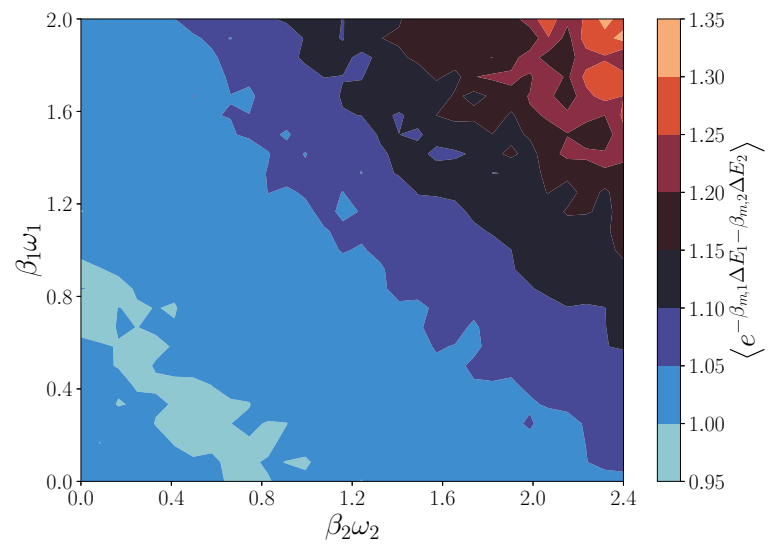

FIG. 13. Measured values of $\left\langle e^{-\beta_{m, 1} \Delta E_{1}-\beta_{m, 2} \Delta E_{2}}\right\rangle$ for the QMC engine design in Fig. 12 as a function of nominal inverse rescaled temperatures $\beta_{1} \omega_{1}, \beta_{2} \omega_{2}$. (a)
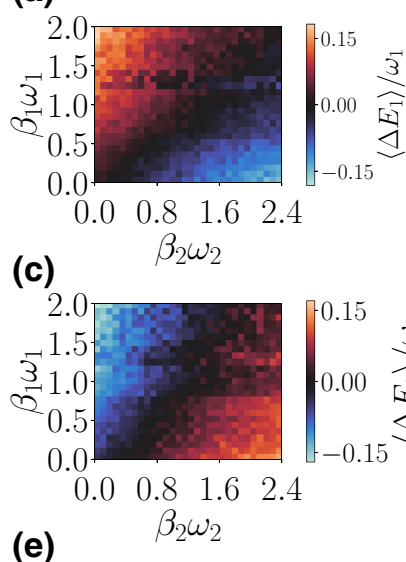

(e)

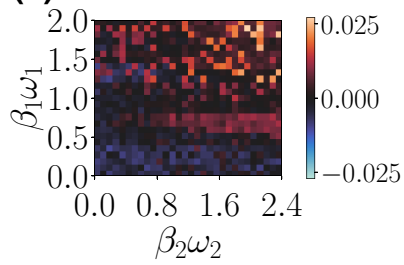

(b)
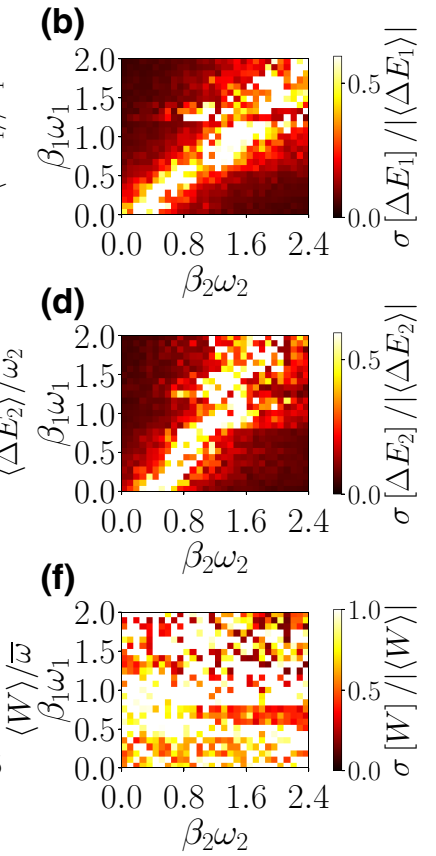

FIG. 14. (a),(c),(e) Measured rescaled energy exchanges $\left\langle\Delta E_{1}\right\rangle / \omega_{1},\left\langle\Delta E_{2}\right\rangle / \omega_{2},\langle W\rangle / \bar{\omega}$, for the QMC engine design in Fig. 12 as a function of nominal inverse rescaled temperatures $\beta_{1} \omega_{1}, \beta_{2} \omega_{2}$. (b),(d),(f) Their relative experimental error.

are smaller than half of the according values measured for the SWAP engine. In our understanding that is due to the considerably more complex circuit used to implement this design, which is expected to be affected by larger noise and energy leaks. That this is in fact the case, can be seen by noticing that relative errors are larger in this case compared to the SWAP engine case.

As in the SWAP case, due to the closeness of the qubits level spacings, cooling was not achieved, and the operation observed, for all values of the nominal inverse rescaled temperatures $\beta_{i} \omega_{i}$ was mostly that of a thermal accelerator. The experimentally observed "phase diagram" is qualitatively similar to that observed for the SWAP engine, only more noisy (not shown). Exactly as in the SWAP engine case, for the implementation of cooling, qubits with appreciably different level spacing are necessary. That however is not a knob that a remote user of the quantum processor can access.

\section{CONCLUSIONS}

With this work we have laid the experimental foundations for the study of nonequilibrium thermodynamic phenomena in quantum processors.

We have put forward a novel ancilla-assisted protocol for the practical implementation of the two-point measurement scheme and have experimentally validated its efficacy on an IBM quantum processor. The method can 
be straightforwardly ported onto other quantum computing platforms. Compared to the interferometric method $[18,19]$, which gives a discrete sampling of the work characteristic function, hence a coarse-grained work probability distribution function, our method gives the work statistics exactly and directly, without the need to perform an inverse Fourier transform. It does accordingly perform much better in terms of Quantum Processing Unit (QPU) time. The price to be paid is that our method requires the initialization of system and ancilla in the TFD state [31,32], which requires an ancilla with the same size as the system, while in the interferometric method the ancilla is a single qubit, regardless of the system size. In comparison with the method of Roncaglia et al. [21], which also gives only a coarse-grained version of the work statistics (1), our method gives it exactly. However, since, our method needs the doubling of the Hilbert space, for a sufficiently large system it would not be implementable at all, while the Roncaglia et al. method, would at least give an approximate solution. This would be the case, for example, if the the system is composed of $N$ qubits, and a total of $K<2 N$ qubits are available on the quantum hardware.

It is worth also stressing that, while for noninteracting or for sufficiently small systems, the task of preparing the TFD state is easily achieved, that is a considerably complex task for large interacting systems. Several proposal for the solution of this problem are available, implying quantum [29,30], classical [33,34], or variational hybrid quantum-classical [31,32] algorithms. Summing up, for sufficiently small systems, the ancilla-assisted method is most effective in terms of precision and QPU time.

We implemented the method on IBM quantum processors and employed it to experimentally corroborate the prediction that the fluctuation theorem is robust to intermediate projective measurements of a generic observable [6,7], which was not experimentally observed so far.

We also demonstrated, for the first time, the implementation of a quantum heat-engine design that is in the limelight of current research, namely the two-qubit and two-stroke SWAP quantum engine. We employed our ancilla-assisted two-point measurement scheme to corroborate the validity of the heat-engine fluctuation theorem, a theoretical prediction that was so far only addressed with the interferometric method [43]. We further verified, for the first time, the validity of the heat-engine fluctuation theorem for the case of a measurement fueled quantum heat engine. That result is at the basis of the mechanism of quantum-measurement cooling [11], whereby a qubit is cooled down by the very act of being measured (along with a second hotter qubit) in an appropriate measurement basis.

For both heat engines implemented, the observed mode of operation was mostly that of a thermal accelerator, namely, physically what happened is that the cold qubit was heated, the hot qubit was cooled down, while energy was spent to make this happen. Interestingly, the same was observed in an experimental study of the thermodynamics of a quantum annealer [26]. The fact that other modes of operations were not observed is a consequence of the fact that the qubits on the IBM quantum processors have very similar resonant frequencies. In order for the engine to work, e.g., as a cooler, qubits with substantially different level spacings are necessary. Our results then, clearly indicate that in order to cool down qubits on a quantum processor, using a SWAP quantum heat engine, it is crucial to equip them with "service" qubits of larger level spacings, that can be used to extract energy from cold "computational" qubits. Such a thermodynamic mechanism could be employed, e.g., to improve the purity of the computational qubits' preparation, by sacrificing the purity of the service qubits.

\section{ACKNOWLEDGMENTS}

We are grateful to Professor P. Hänggi and Professor P. Talkner for their useful comments and remarks. We acknowledge the use of IBM Quantum services for this work [12]. The views expressed are those of the authors, and do not reflect the official policy or position of IBM or the IBM Quantum team. In this paper we used ibmq_belem, which is one of the IBM Quantum Falcon r4 Processors. Andrea Solfanelli and Alessandro Santini acknowledge that their research has been conducted within the framework of the Trieste Institute for Theoretical Quantum Technologies (TQT).

[1] Y. Alexeev et al., Quantum computer systems for scientific discovery, PRX Quantum 2, 017001 (2021).

[2] J. Goold, M. Huber, A. Riera, L. del Rio, and P. Skrzypczyk, The role of quantum information in thermodynamics - a topical review, J. Phys. A: Math. Theo. 49, 143001 (2016).

[3] S. Deffner and S. Campbell, in Quantum Thermodynamics (Morgan \& Claypool Publishers, San Rafael, CA, 2019).

[4] J. Millen and A. Xuereb, Perspective on quantum thermodynamics, New J. Phys. 18, 011002 (2016).

[5] M. Campisi, P. Talkner, and P. Hänggi, Fluctuation Theorem for Arbitrary Open Quantum Systems, Phys. Rev. Lett. 102, 210401 (2009).

[6] M. Campisi, P. Talkner, and P. Hänggi, Fluctuation Theorems for Continuously Monitored Quantum Fluxes, Phys. Rev. Lett. 105, 140601 (2010).

[7] M. Campisi, P. Talkner, and P. Hänggi, Influence of measurements on the statistics of work performed on a quantum system, Phys. Rev. E 83, 041114 (2011). 
[8] M. Campisi, Fluctuation relation for quantum heat engines and refrigerators, J. Phys. A: Math. Theo. 47, 245001 (2014).

[9] The very first experiment has been reported as we write, see Ref. [43].

[10] M. Campisi, J. Pekola, and R. Fazio, Nonequilibrium fluctuations in quantum heat engines: Theory, example, and possible solid state experiments, New J. Phys. 17, 035012 (2015).

[11] L. Buffoni, A. Solfanelli, P. Verrucchi, A. Cuccoli, and M. Campisi, Quantum Measurement Cooling, Phys. Rev. Lett. 122, 070603 (2019).

[12] IBM quantum, https:/quantum-computing.ibm.com/ (2021).

[13] H. Tasaki, Jarzynski relations for quantum systems and some applications, ArXiv:cond-mat/0009244 (2000).

[14] J. Kurchan, A quantum fluctuation theorem, ArXiv:condmat/0007360 (2000)

[15] P. Talkner, E. Lutz, and P. Hänggi, Fluctuation theorems: Work is not an observable, Phys. Rev. E 75, 050102(R) (2007).

[16] M. Campisi, P. Hänggi, and P. Talkner, Colloquium. Quantum fluctuation relations: Foundations and applications, Rev. Mod. Phys. 83, 771 (2011).

[17] M. Campisi, P. Hänggi, and P. Talkner, Erratum: Colloquium: Quantum fluctuation relations: Foundations and applications [Rev. Mod. Phys. 83, 771 (2011)], Rev. Mod. Phys. 83, 1653(E) (2011).

[18] R. Dorner, S. R. Clark, L. Heaney, R. Fazio, J. Goold, and V. Vedral, Extracting Quantum Work Statistics and Fluctuation Theorems by Single-Qubit Interferometry, Phys. Rev. Lett. 110, 230601 (2013).

[19] L. Mazzola, G. De Chiara, and M. Paternostro, Measuring the Characteristic Function of the Work Distribution, Phys. Rev. Lett. 110, 230602 (2013).

[20] T. B. Batalhão, A. M. Souza, L. Mazzola, R. Auccaise, R. S. Sarthour, I. S. Oliveira, J. Goold, G. De Chiara, M. Paternostro, and R. M. Serra, Experimental Reconstruction of Work Distribution and Study of Fluctuation Relations in a Closed Quantum System, Phys. Rev. Lett. 113, 140601 (2014).

[21] A. J. Roncaglia, F. Cerisola, and J. P. Paz, Work Measurement as a Generalized Quantum Measurement, Phys. Rev. Lett. 113, 250601 (2014).

[22] F. Cerisola, Y. Margalit, S. Machluf, A. J. Roncaglia, J. P. Paz, and R. Folman, Using a quantum work meter to test non-equilibrium fluctuation theorems, Nat. Commun. 8, 1241 (2017).

[23] A. Smith, Y. Lu, S. An, X. Zhang, J.-N. Zhang, Z. Gong, H. T. Quan, C. Jarzynski, and K. Kim, Verification of the quantum nonequilibrium work relation in the presence of decoherence, New J. Phys. 20, 013008 (2018).

[24] S. An, J.-N. Zhang, M. Um, D. Lv, Y. Lu, J. Zhang, Z.Q. Yin, H. T. Quan, and K. Kim, Experimental test of the quantum Jarzynski equality with a trapped-ion system, Nat. Phys. 11, 193 (2015).

[25] L. Bassman, K. Klymko, N. M. Tubman, and W. A. de Jong, Computing free energies with fluctuation relations on quantum computers, ArXiv:2103.09846 (2021).
[26] L. Buffoni and M. Campisi, Thermodynamics of a quantum annealer, Quantum Sci. Technol. 5, 035013 (2020).

[27] S. Hernández-Gómez, N. Staudenmaier, M. Campisi, and N. Fabbri, Experimental test of fluctuation relations for driven open quantum systems with an NV center, New J. Phys. 23, 065004 (2021).

[28] Since the system is prepared in each run of the experiment in a specific energy eigenstate, the initial energy measurement become superfluous.

[29] D. Poulin and P. Wocjan, Sampling from the Thermal Quantum Gibbs State and Evaluating Partition Functions with a Quantum Computer, Phys. Rev. Lett. 103, 220502 (2009).

[30] A. Riera, C. Gogolin, and J. Eisert, Thermalization in Nature and on a Quantum Computer, Phys. Rev. Lett. 108, 080402 (2012).

[31] J. Wu and T. H. Hsieh, Variational Thermal Quantum Simulation via Thermofield Double States, Phys. Rev. Lett. 123, 220502 (2019).

[32] D. Zhu, S. Johri, N. M. Linke, K. A. Landsman, C. Huerta Alderete, N. H. Nguyen, A. Y. Matsuura, T. H. Hsieh, and C. Monroe, Generation of thermofield double states and critical ground states with a quantum computer, Proc. Natl. Acad. Sci. USA 117, 25402 (2020).

[33] S. R. White, Minimally Entangled Typical Quantum States at Finite Temperature, Phys. Rev. Lett. 102, 190601 (2009).

[34] E. M. Stoudenmire and S. R. White, Minimally entangled typical thermal state algorithms, New J. Phys. 12, 055026 (2010).

[35] M. A. Nielsen and I. L. Chuang, Quantum Computation and Quantum Information: 10th Anniversary Edition (Cambridge University Press, Cambridge, England, 2010).

[36] C. Elouard, D. A. Herrera-Martí, M. Clusel, and A. Auffèves, The role of quantum measurement in stochastic thermodynamics, npj Quantum Inf. 3, 9 (2017).

[37] J. Yi, P. Talkner, and G.-L. Ingold, Approaching infinite temperature upon repeated measurements of a quantum system, Phys. Rev. A 84, 032121 (2011).

[38] G. Giachetti, S. Gherardini, A. Trombettoni, and S. Ruffo, Quantum-heat fluctuation relations in three-level systems under projective measurements, Condens. Matter 5, 17 (2020).

[39] S. Gherardini, G. Giachetti, S. Ruffo, and A. Trombettoni, Thermalization processes induced by quantum monitoring in multi-level systems, arXiv:2012.15216 (2020).

[40] B. Misra and E. C. G. Sudarshan, The zeno's paradox in quantum theory, J. Math. Phys. 18, 756 (1977).

[41] A. Peres, Zeno paradox in quantum theory, Am. J. Phys. 48, 931 (1980).

[42] D. Andrieux, P. Gaspard, T. Monnai, and S. Tasaki, The fluctuation theorem for currents in open quantum systems, New J. Phys. 11, 043014 (2009).

[43] T. Denzler, J. F. G. Santos, E. Lutz, and R. Serra, Nonequilibrium fluctuations of a quantum heat engine, arXiv:2104.13427 (2021).

[44] S. Lloyd, Quantum-mechanical maxwell's demon, Phys. Rev. A 56, 3374 (1997). 
[45] H. T. Quan, Y. X. Liu, C. P. Sun, and F. Nori, Quantum thermodynamic cycles and quantum heat engines, Phys. Rev. E 76, 031105 (2007).

[46] A. E. Allahverdyan, R. S. Johal, and G. Mahler, Work extremum principle: Structure and function of quantum heat engines, Phys. Rev. E 77, 041118 (2008).

[47] A. M. Timpanaro, G. Guarnieri, J. Goold, and G. T. Landi, Thermodynamic Uncertainty Relations from Exchange Fluctuation Theorems, Phys. Rev. Lett. 123, 090604 (2019).
[48] R. Uzdin and R. Kosloff, The multilevel four-stroke swap engine and its environment, New J. Phys. 16, 095003 (2014).

[49] A. Solfanelli, M. Falsetti, and M. Campisi, Nonadiabatic single-qubit quantum Otto engine, Phys. Rev. B 101, 054513 (2020).

[50] A. Solfanelli, L. Buffoni, A. Cuccoli, and M. Campisi, Maximal energy extraction via quantum measurement, J. Stat. Mech.: Theory Exp. 2019, 094003 (2019). 